\title{
Recognizing Physical Activities Using Wii Remote
}

\author{
Ali Mahmood Khan
}

\begin{abstract}
Physical activity is a major part of the user's context for wearable computing applications. System should be able to acquire the user's physical activities using body worn sensors. We proposed to use the Wii-remote which is a readymade, light weight, small and easy to use device for identifying basic physical activities like lying, sitting, walking and running using decision tree classifier.
\end{abstract}

Index Terms-Physical activities, accelerometer sensor, decision tree classifier.

\section{INTRODUCTION}

One of the major scientific undertakings over the past few years has been exploring the interaction between humans and machines in the mobile environment. Research projects have brought forth various possibilities of interaction between humans and computers. Our daily lives are becoming more and more pervasive: we now have very smart devices with high computational power.

Recognizing human physical activities with body worn sensors is not a new research field; lot of research has been done in this area. We can identify the users' physical movements using body movement suit [1], we do have other research projects where researchers identify the users' physical activities using some sensors like [2]-[7].

We want to develop a physical activity recognition system using minimum amount of sensors which should be able to identify the basic physical activities like lying, sitting, walking, and running.

In our research we want to prove that it is possible by using a 3D accelerometer to specifically identify the four main physical activities i.e. lying, sitting, walking and running for a specific user.

\section{RELATED WORK}

Since everyone has a different body structure, data collected by an activity measuring sensor would also be different. The device should be able to learn and adapt from an individual's physical activities. We want to identify the users' basic physical activities using only one accelerometer in our research.

Researchers have identified the various physical activities using wearable sensors like sitting[2], [5]-[7], standing[2]-[7], lying[7], walking[2]-[7], climbing stairs[2][7], running[4], [6]-[7], cycling[4], [7], strength-training[7] etc.

Manuscript received on August 20, 2012; revised November 28, 2012.

Ali Mahmood Khan is with University of Bremen TZi, Bremen, Germany (e-mail: akhan@tzi.de).
However researchers have identified several activities of interest but they used more than one sensor for this purpose. For example, researchers identified around 20 activities using 5 sensor boards. They identified Walking, Walking carrying items, Sitting \& relaxing, Working on computer, Standing still, Eating or drinking, watching TV, Reading, Running, Bicycling, Stretching, Strength-training, Scrubbing, Vacuuming, Folding laundry, Lying down \& relaxing, Brushing teeth, Climbing stairs, Riding elevator and Riding escalator using Decision Table, IBL, C4.5 and Naive Bayes algorithms. They placed sensors on the limb positions and on the right hip [7]. Similarly researchers identified 12 activities using 3 sensor boards, they identified Sitting, Standing, Walking, Walking up stairs, Walking down stairs, Riding elevator down, Riding elevator up, Brushing Teeth[2], researchers identified 3 activities; Walking, Climbing stairs and Descending stairs using 9 tilt switches using K-means clustering and Brute force algorithms, these sensors were worn just above the right knee[3].

In our work, we want to use only one 3D sensor to identify few activities that is Sitting, Lying, Walking and Running. That is why we used accelerometer sensor of the Wii remote device because it is a readymade device and we did not want to spend our time for the hardware design. We implemented the application in Java which used to log the accelerometers values of Wii remote [8] into the system.

\section{HYPOTHESIS}

The acceleration measured by a 3 axis accelerometer $(\mathrm{X}, \mathrm{Y}, \mathrm{Z})$ at a specific point (backbone), indicates which activity the person is performing (lying, sitting, walking and running), using decision tree classifier.

\section{EXPERIMENTAL Methodology}

This section describes the approach we adopted. It introduces the experimental set-ups, including the number and placement of sensors and the gathered data in detail.

\section{A. Experimental Setup}

In order to identify the physical activities, we used a Wii remote [8] during the lab testing and asked our testers to wear it around their backs as shown in Fig. 1.

The Wii remote was connected to the laptop via blue-tooth. Our application which was running on the laptop and used to $\log$ the three accelerations $(x, y, z)$ for the off-line analysis.

1) Setup 1: For our initial tests we recruited five testers. We noticed that it was not easy for the testers to place the device in a fixed position for the whole duration of the tests by just using a belt, sometimes the device dropped down during the tests. It was important to stick the Wii 
remote on the same position during the experiment according to our hypothesis. To rectify the problem we built a case for the Wii remote, put it into a case and fixed to a belt as shown in Fig. 2. This case was reliable and it was fixed on the same position of the testers' body during the whole experiment.

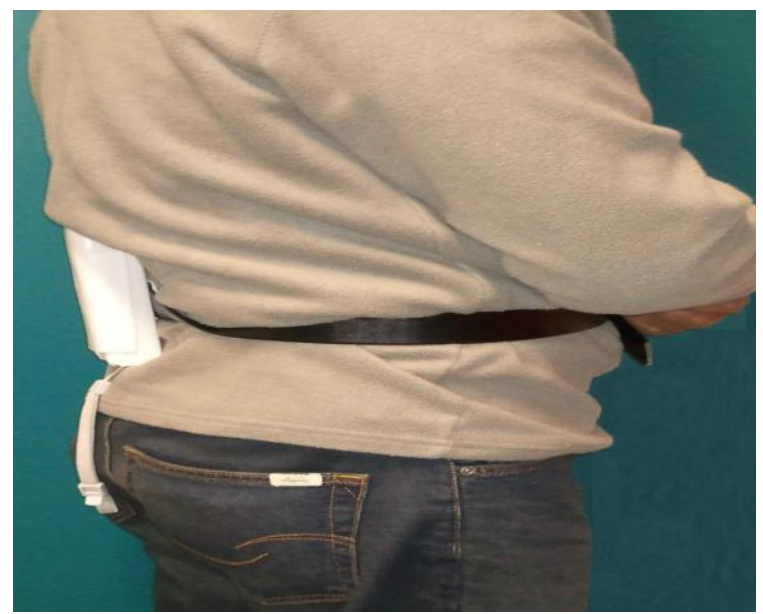

Fig. 1. Subject with wii remote

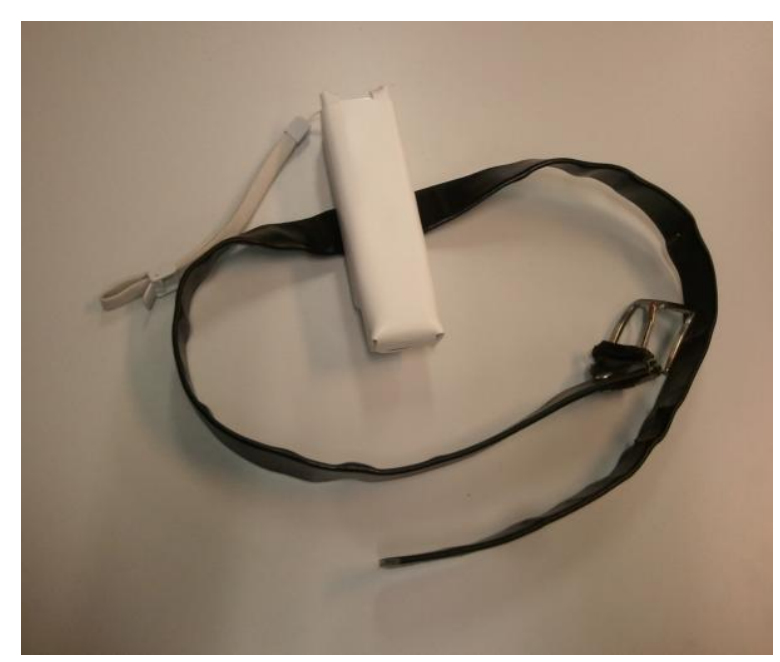

Fig. 2. Wii remote case

2) Setup-2: We recruited 24 male testers for the second setup. Data from three testers was discarded because their accelerometer's data was not logged completely due to Bluetooth's connectivity problem. The age of the remaining testers ranged from 21 to 34 ( mean 26.38, sd 4.18) and ranged in BMI(body mass index)[10] from 19.7 to 38.4 (mean 25.65, SD 4.28). We asked our testers to perform the activities (lying, sitting, walking and running) twice. Each activity took them one minute. We continuously observed our testers while conducting the experiments and noted down the activity and starting/ending time of each activity. It was a lab testing and our testers had almost the same position while lying and sitting. For walking/running; they used to walk/run on the corridor from one end to another end with constant speed.

\section{DAta Gathering Results}

Basically, our testers were supposed to do four physical activities (lying, sitting, walking and running) and each activity took those sixty seconds to complete. The Wii remote reads around 100 values $(X, Y, Z)$ per second. We got (100X60X4) 24,000 instances for each data-set.

At the end we had two data sets from each tester. We separated datasets with the help of time stamp which was also mentioned it in the log file. Each dataset contained all the required physical activities. One dataset represented the training data and other dataset was used for test data.

We applied the decision tree 'J48' classifier using weka toolkit [9] on the training dataset. It correctly classified instances from $92.74 \%$ to $98.9 \%$ (average: $96.61 \%$, SD: 1.63). We built the model for each tester separately. Later, we applied the model on the test data in order to predict the physical activities and compared the machine's predictions with the actual values.

We got 100 values $(\mathrm{X}, \mathrm{Y}, \mathrm{Z})$ from the Wii remote for each second, we also got 100 predictions for each second. We wanted to have a single prediction for each second.

During the training of each tester's data, the classifiers successfully classified $\mathrm{X} \%$ instances. This ' $\mathrm{X}$ ' figure was used as our threshold for the confidence values of the classifier during testing, implying all instances which had a lower confidence value than our threshold were discarded. From this reduced subset of instances, the activity with the maximum number of instances was chosen. This resulted in a single value for each second, instead of the 100 values that are received from the Wii remote every second, leading to a much easier analysis of the experiment. Then these single values were compared with the actual values of physical activity to realize the accuracy of our test.

The above description is also explained in the pseudo code:

Correctly Classified Instances : $\mathrm{X}=$ decision tree(training data) testing data(a[1 to 100$])$

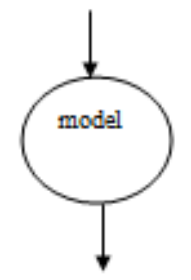

ans[1 to 100$]=\operatorname{predicted} \operatorname{data}(1$ to 100$)$ for $i=1$ to 100

\{

$$
\begin{aligned}
& \text { if ans[i].confedence level }>=X \\
& \text { if ans(i) = 'lying' } \\
& \text { \{ ilying++ } \\
& \text { \} } \\
& \text { if } \operatorname{ans}(\mathrm{i})=\text { 'Sitting' } \\
& \{ \\
& \text { iSitting++ } \\
& \text { if ans(i) = 'Walking' } \\
& \{
\end{aligned}
$$


\}

if ans(i) = 'Running'

\{

\}

iRunning++

\}

Activity per second= MAX (iLying, iSitting, iWalking, iRunning)

The physical activity with the maximum number of instances was chosen for each second.

TABLE I: PREDICTED RESULTS FOR 21 TESTERS

\begin{tabular}{|c|c|c|c|c|}
\hline & LYING & SITTING & WALKING & RUNNING \\
\hline MIN & $98.83 \%$ & $90.77 \%$ & $71.19 \%$ & $98.31 \%$ \\
\hline MAX & $100.00 \%$ & $100.00 \%$ & $100.00 \%$ & $100.00 \%$ \\
\hline AVG & $99.31 \%$ & $99.31 \%$ & $96.12 \%$ & $99.92 \%$ \\
\hline SD & $2.55 \%$ & $2.11 \%$ & $9.57 \%$ & $0.37 \%$ \\
\hline
\end{tabular}

The results show that our system was able to predict the physical activities with good accuracy.

\section{CONCLUSION}

Wii remote's accelerometer sensor was able to recognize a high percentage of the physical activities with the help of the decision tree classifier. Results have shown that one 3D accelerometer sensor is enough for identifying a few physical activities (Sitting, Lying, Walking and Running). For every user, the system needs to be trained with the sensor data so that it would be able to predict the physical activities using Wii remote. This prototype is a proof of the concept and our results show that only one $3 \mathrm{D}$ accelerometer sensor can identify the above mentioned physical activities of any kind of BMI (body mass index) and age. Accelerometer sensor has to be fixed properly on the backbone of the tester in order to predict the tester's movements successfully.

\section{FUTURE WORK}

We will put the accelerometer sensor on other parts of the body in order to identify some other physical activities and we will use it for online machine learning.

\section{ACKNOWLEDGMENT}

I am sincerely thankful to my supervisor, Prof. Dr. Michael Lawo, TZi who helped me at every stage during my research. He helped me in clearing all my doubts and increased my knowledge through his valuable suggestions and inputs. I am very thankful to my testers, for voluntarily testing my setup.

\section{REFERENCES}

[1] Xsens MVN. (9th September, 2010) [Online]. Available: http://www.xsens.com/en/general/mvn

[2] J. Lester, T. Choudhury, and G. Borriello, "A practical approach to recognizing physical activities," Lecture Notes in Computer Science, vol. 3968, 2006, pp. 1-16.

[3] K. V. Laerhoven and A. K. Aronsen, "Memorizing what you did last week: Towards detailed actigraphy with a wearable sensor," Distributed Computing Systems Workshops, 2007, ICDCSW'07, presented at 27th International Conference on, 2007, pp. 47-47.

[4] T. Choudhury et al., "The mobile sensing platform: An embedded activity recognition system," IEEE Pervasive Computing, 2008, pp. $32-41$.

[5] N. Kern, B. Schiele, and A. Schmidt, "Multi-sensor activity context detection for wearable computing," Lecture Notes in Computer Science, 2003, pp. 220-234.

[6] U. Maurer et al., "Location and Activity Recognition Using eWatch: A Wearable Sensor Platform," Lecture Notes in Computer Science, vol. 3864, 2006, pp. 86.

[7] L. Bao and S. S. Intille, "Activity recognition from user-annotated acceleration data," Lecture Notes in Computer Science, 2004, pp. 1-17.

[8] Wii. (9th September, 2010). [Online]. Available: http://www.nintendo.com/wii

[9] M. Hall, E. Frank, G. Holmes, B. Pfahringer, P. Reutemann, and I. H. Witten, "The WEKA Data Mining Software: An Update," SIGKDD Explorations, 2009, vol. 11, no. 1.

[10] Body Mass Index. (27th January, 2011). [Online]. Available: http://www.nhlbisupport.com/bmi/

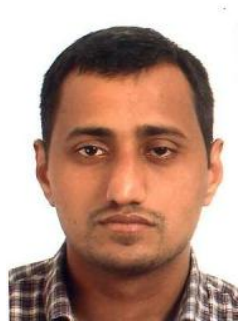

Ali Mahmood Khan was born on 30th of May, 1982, in Lahore, Pakistan. He is pursuing $\mathrm{PhD}$ in the field of Wearable computing from Bremen University, Germany. His major is Human computer Interaction and MSc in Digital Media (Computer Sciences) from Hochschule Bremen, Germany in March 2009. He majored in Mobile HCI and obtained Bachelor in Computer Sciences from OPSTeC, Lahore, Pakistan in August 2004. He is working as a scientific researcher at Bremen University, Germany and as a software developer at Verivox, Heidelberg and as a lecturer at the Pakistani university UMT (http://www.umt.edu.pk/). 